\title{
VISIBILITY ANALYSIS OF HUGE OUTDOOR ADVERTISEMENTS ALONG GUADALUPE BRIDGE IN EDSA HIGHWAY FROM STRUCTURE-FROM-MOTION PHOTOGRAMMETRY
}

\author{
M. N. Manansala, R. M. Ong, K. A. Vergara \\ Department of Geodetic Engineering, University of the Philippines, Diliman, Quezon City, Philippines \\ nathmanansala@gmail.com, remong17@gmail.com, kpvergara@up.edu.ph
}

KEY WORDS: Structure from motion, Close-Range Photogrammetry, Visibility Analysis, 3D GIS, Outdoor Advertising

\begin{abstract}
:
When it comes to business and marketing, huge outdoor advertising is considered as one of the best ways by contributing largely in disseminating information about a product, service or even raise awareness. With commuters or the people riding in a moving car as its target audience, the placement of advertising materials is very crucial since it should be visible and must deliver its message in a short span of time. This study tests the methodology of gathering data using action camera and DSLR mounted and situated on a moving vehicle, utilizing structure from motion techniques, to extract the geometry of the billboards from the point cloud generated from structure-from-motion as acquired from camera videos that would be used to represent these billboards in the three-dimensional space. These extracted geometries would be used for visibility analysis from a passenger's point of view by assessing the percentage of visible content and logos of each billboard from each point of observation along the path of a moving vehicle. The results of this study are nine sets of mean percent visibilities and raster representations that show the mean percent visibility of the billboards as viewed from the road of interest. To assess product placement effectiveness of the billboards, visibility percentage of the product logos contained in the nine billboards was also obtained.
\end{abstract}

\section{INTRODUCTION}

\subsection{Background of the Study}

In advertising, there are numerous means and ways for communicators to reach out to their target audiences. A communication tool that has been growing in popularity is the outdoor advertising. These advertisements have the unique ability to display messages 24 hours a day, seven days a week. Drivers and commuters pass through the same messages numerous times, which makes this kind of media effective. (Lithgow, 1999). Various kinds of these huge advertisements are commonly placed in the urban areas and cities.

Here in the Philippines, in Metro Manila, the center of urbanization, these kinds of advertisements are seen everywhere especially on busy main thoroughfares. Like any other cities, Metro Manila is subject to various elements that could affect the effectiveness of these huge advertisements. With the billboards' proximity to fixed structures, the physical placement of the billboards is one the first considerations. The placement of advertising materials has been treated as one of the main considerations of the advertising companies on the effectiveness of the advertisements. (Lucas, et al, 1997). To assess this, the visibility of these billboards from a moving vehicle passenger or a passerby is considered. If these billboards are not effectively by commuters, it will not serve its purpose. Moreover, this primarily targets the commuters or the people riding in a car. Therefore, billboards are considered placed effectively if the target audience can easily see or get the thought of the advertisements in a short span of time. (Lithgow,1999).

Also, the placement of logos and the catchy phrases also contribute into the effectiveness of these. Once the driver or passenger notices these huge billboards but can't remember about the logo or brand name of the product or service it conveys, these billboards may be rendered useless. These logos and phrases create identity of the products, thus, shall be visible as possible. (Gudis, 2004).

Several studies have ventured into the analysis of huge outdoor advertisements through photogrammetry and GIS (Aydin, et al, 2008; Chmielewski, et al, 2017; Chmielewski, et al, 2015). However, few to none have ventured the use of structure from motion photogrammetry as a tool for visibility analysis.

From multiple overlapping images as taken in different views together with camera parameters, Structure from Motion (SfM) algorithms take these inputs to reconstruct 3D positions of points and camera poses in a common coordinate system. (Moulon, et al, 2012). Through structure-from-motion photogrammetry, the 3D geometry of billboards can be estimated to assess the visibility of the billboards along a road of interest. In this study, the effectiveness of billboards as assessed through its visibility along Guadalupe Bridge in EDSA Highway will be analyzed.

\subsection{Study Area}

EDSA or Epifanio de los Santos Avenue is a 24-kilometer road that passes through the six of the 16 cities of Metro Manila, namely Caloocan City, Quezon City, San Juan City, Mandaluyong City, Makati City, and Pasay City. It connects the Northern and the Southern hemisphere of Metro and Greater Manila. It passes through Makati, titled as the "Financial Center of the Philippines", wherein The Philippine Stock Exchange is centered and most of the top corporations and institutions' main offices are located. This is where the majority of the working class travel from North to South and vice versa.

More specifically, since this research is devoted to assessing the visibility effectiveness of the billboards along EDSA Highway, the study area has been narrowed down to Guadalupe Bridge 
wherein billboards are rampantly congested and unstoppably rising in number. The said bridge connects the cities of Makati and Mandaluyong with a length of approximately 114.44 meters, while its width is approximately 18.70 meters, measuring 3.35 meters for every lane. Since there are 5 lanes on the bridge, but only the 2-lane part is to be considered, the width of the road is narrowed down to 6.70 meters.

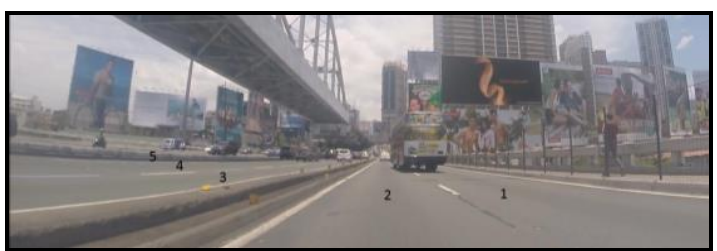

Figure 1. Lanes along the bridge; numbered from 1-5 where the first two lanes are the only lanes to be considered

The road on the Guadalupe Bridge is divided into 5 lanes but are still partitioned to 3 to 2 lanes shown in the figure above, captured from the data acquired. The 2-lane road, at the rightmost part, is the part of the bridge where the observer's (passenger's) point of view will be defined.

\subsection{Objectives}

This study aims to test the methodology of assessing the effectiveness of huge outdoor advertisements from structurefrom-motion photogrammetry outputs. The geometries as derived from the point cloud generated shall be used to obtain the visibility of billboard advertisements from viewpoints along the northbound side of Guadalupe Bridge in EDSA highway, and to assess the visibility percentage of the logos, contained in the billboard advertisements.

\subsection{Scope and Limitations}

Data acquisition was done on a Sunday morning, approximately 9:00 am to 10:00 am, when the traffic is less congested than when on a weekday to avoid being stuck in the traffic and to be able to acquire data under a certain set of circumstances.

A sedan-type vehicle was used to conduct the data acquisition and will set a standard height of eye level of a passenger to be $1.08 \mathrm{~m}$ from the road. Furthermore, this study only considered the northbound section of EDSA along Guadalupe Bridge, specifically along the path railway of MRT. Since the contents of the billboard change through time especially for LED-type billboards, the contents of the billboards at the time of acquisition were only considered for assessment. In the case of the determination of visibility, only the sets of point cloud generated from the processing will only be the basis for the generation of road, billboard, and obstruction geometries.

\section{METHODOLOGY}

\subsection{General Workflow}

The figure below describes the general steps done in conducting this research.

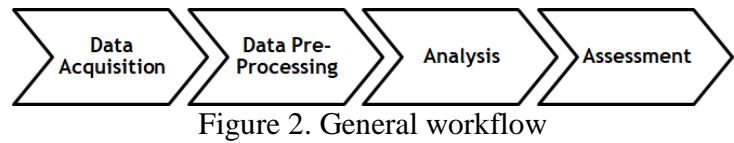

Figure 2. General workflow

\subsection{Data Acquisition}

The video recordings were obtained using $(a, b)$ two DSLR cameras that were oriented at two different directions, however directed to two dissimilar directions, both are held at the same height. Another acquisition was made with a GoPro camera, but this time, the camera was mounted on the front hood of the vehicle. A (c) GoPro Hero 3+ camera was mounted on the front hood of the vehicle via a car suction mount. There were two sets of recorded videos for this acquisition: angled such that only the road can be recorded and angled such that the road and the billboards are captured.

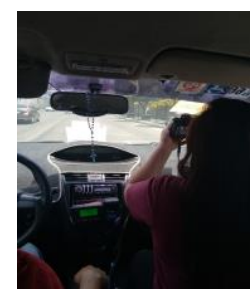

(a)

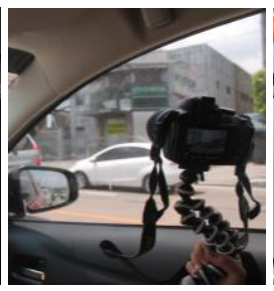

(b)

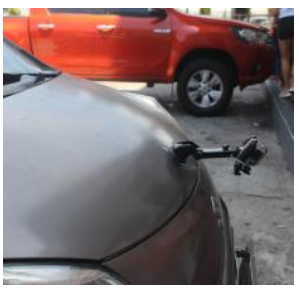

(c)
Figure 3: Video acquisition: (a, b) DSLR Camera held inside the car; and (c) GoPro camera mounted at hood of car

Nine billboards were identified and considered for analysis. The figure below shows the billboards and are named as follows.

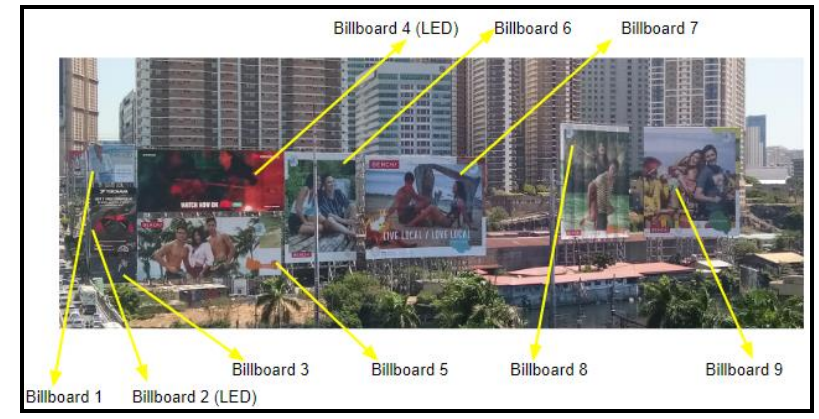

Figure 4. Billboards considered

\subsection{Data Pre-processing}

\subsubsection{Point Cloud Generation}

The first in the pre-processing is the extraction of images where a third-party software was used, the Free Video to JPG Converter to have the videos extracted into images. Shown below are the number of photos extracted per camera used:

\begin{tabular}{|c|c|}
\hline Camera & Number of images extracted \\
\hline $\mathrm{a}$ & 457 \\
\hline $\mathrm{b}$ & 342 \\
\hline $\mathrm{c}$ & 532 \\
\hline
\end{tabular}

Table 1. Images extracted

These images were processed in Agisoft Photoscan using the default settings to generate three sets of point clouds-one from each camera source.

\subsubsection{Point Cloud Processing}

The three sets of points clouds generated separately were aligned, georeferenced, and cleaned using CloudCompare. CloudCompare is an opensource 3D point cloud editing and processing software originally designed for dense $3 \mathrm{D}$ point 
cloud comparison but extended its capability to various point cloud processing algorithms (CloudCompare Project Team, 2015). This software made the merging and cleaning of the three sets of point clouds possible. The merging was done by using the Align tool by picking (at least 4) equivalent point pairs while the cleaning was done by using the segment tool. Cleaning the point clouds from unwanted noises was necessary to clearly define the geometries of the billboards as well as obstructions. For a more convenient processing and analysis later on, the cleaned point cloud was reoriented such that the road's direction is directly facing the north and that the properly merged and scaled final point cloud was split and exported into 3 sets of LAS files having separate files for the billboards, road, and obstructions. Moreover, for the analysis of logo placement, from the billboards LAS file, the logos on each billboard were segmented such that the logos will have a separate LAS file.

\subsubsection{Billboard-road-obstruction geometry}

From these sets of point cloud, a billboard-road-obstructions vector-based environment was generated from the final point cloud. To achieve this, the three sets of LAS files were digitized according to these categorical features: billboards, road, and obstructions. Through ArcScene, LAS files were converted into multipoint, to manually digitize the extent of the road, billboards, and obstructions to produce three vector files.

\subsubsection{Observer and Target Points}

Before proceeding with the visibility analysis, after the geometry of the billboards were extracted by digitizing, observer points were generated by dividing the road polygon into 100 equal portions, using Fishnet ( 2 columns to represent 2 lanes of road in EDSA and 50 rows for the length of the road) and creating a point at its centroid. The elevation of this point was added with the $1.08 \mathrm{~m}$ to simulate the visibility from a passenger's point-of-view. Apart from creating 100 observer points, 100 equally-spaced target points, positioned on the surface of the digitized billboards, were also created for every billboard. Therefore, there were a total of 900 target points for each observer point on the road. On the other hand, to define the target points for the logos, the extents of the logos of the billboards were intersected with the 100 target points on every billboard, to select the $\mathrm{n}$ number of target points per billboard.

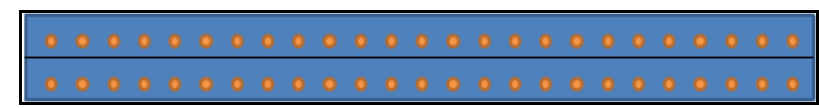

(a)

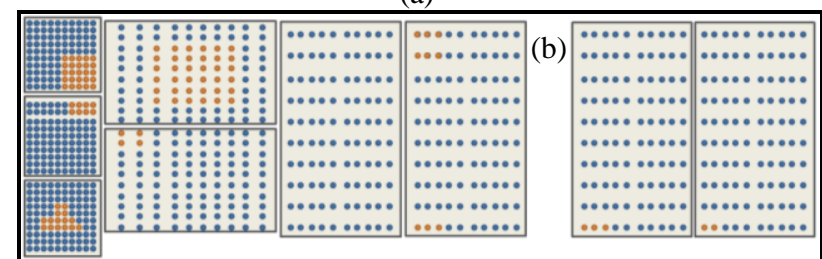

Figure 5: Rough diagram of observer and target points: (a) Observer points along left and right lane of road; and (b) Target points per billboard (blue and orange); Target points of logos (orange)

\subsection{Analysis}

To define the connection of the observer points and the target points, Construct Sightline tool was used in ArcScene. This requires the observer points, the target features, observer height and target height. The observer and target heights were computed by adding $\mathrm{Z}$ information on every point. This tool also adds direction attribute information about the sightline from each of the observer point to each of the target points.

In the determination of the visible target points in the billboards from the observer points, Intervisibility Tool was utilized. The constructed sightlines together with the obstruction features were the inputs for this tool. The obstructions comprise of billboard features with the exception of the billboard being observed and the initially created obstruction polygon from the point clouds. The Intervisibility tool added a new field in the attribute table of the sightlines. It would only give two values 0 and 1 , for not visible and visible, respectively. In order to sort the visibility on every observer point, field calculator was used to filter out the sightlines visible (with field value $=1$ ) from the limitations of a human eye along the road of interest such that the vertical angle ranges from $-70^{\circ}$ to $60^{\circ}$ and that the azimuth ranges from $0^{\circ}$ to $90^{\circ}$ (left side of field of view not considered anymore).

\subsection{Assessment}

\subsubsection{Mean Percent Visibility (MPV)}

The overall visibility of the billboards along the road is tested and measured here. The MPV or Mean Percent Visibility of each billboard was computed based on Equation 1 below:

$$
M P V=\frac{s_{\text {vin }}}{s_{\text {Lur }}} \cdot 100 \%
$$

Where

$$
\begin{aligned}
& S_{\text {vis }}=\text { total number of visible targets } \\
& S_{\text {tar }}=\text { total number of possible targets }
\end{aligned}
$$

For each billboard, there are a total of 10,000 possible targets and the total number of visible targets was determined by getting the sum of all visible targets from each observer point. MPV was determined for the left lane, right lane, and for the whole extent of road.

\subsubsection{Visibility Raster of the Billboards}

For each of the 100 observer points along, the number of visible target points were determined for each billboard. A maximum value of 100, a perfect visibility, can be assigned to an observer point. With a percent visibility assigned to each observer point, visibility raster representations were produced by using these values of observer points as sample points to interpolate the visibility surface along the road using IDW. There would be nine (9) visibility raster files which would be represented with color ramp from red ( $0 \%$ visibility) to green ( $100 \%$ visibility). There were 10 classifications made to normalize the percent visibility into 10 breaks at $10 \%$ increments. This is also to be able to compare the visibility of the billboards at certain parts of the road. Comparing the 9 visibility raster representations would give an idea at what certain parts of the road do some or all contents of billboards are visible.

\subsubsection{Mean Logo Percent Visibility (MLPV)}

To assess the effectivity of the placement of the logos of the products contained in the billboards, The Mean Logo Percent Visibility was obtained. This was computed using the same equation 1; however, in this case, the total number of possible targets are different since only those target points covered by the extent of the logo were considered for visibility analysis. 


\subsubsection{Validation}

For the validation of the results, the researchers conducted a survey using Google Forms. At the beginning of the survey, three videos were attached, and respondents were asked to watch the videos before proceeding with the survey. The order of numbering of the billboards were also illustrated in the survey. The videos consisted of sample video recordings that was obtained during the data acquisition done by the researchers. Videos 1 \& 2 were captured using DSLR Canon $1100 \mathrm{D}$ and were shown to be able to represent the standard vision of a human eye seated at the passenger's seat of a moving sedan-type vehicle. The first video was captured from a moving vehicle situated in the right lane of the road. The second video was taken while in a moving vehicle passing through the left lane of the road.

The first part of the survey is the Percent Visibility. In this section, the respondents were asked what percent each billboard been visible throughout the duration of the video (passing through Guadalupe Bridge). The choices were 0\% $100 \%$ at increments of 10 . The next part of the survey is the Logo Placement Assessment. In this part, the billboards were divided into 9 sections and were labeled from A to I as shown in the next figure. The respondents were then asked which box they have remembered seeing the logos placed. This was also done for all the nine billboards, despite that the 2 billboards are LED boards
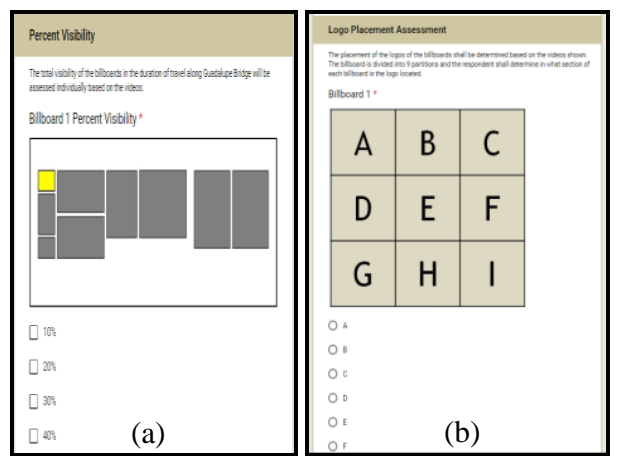

Figure 6: Validation surveys. (a) Percent Visibility Survey; (b)

Logo Placement Assessment

\section{RESULTS AND DISCUSSION}

\subsection{Point cloud}

From the images extracted from the video acquisition, sets of points clouds were generated as shown in Figure 7: From these sets of point clouds, the aligned and cleaned point cloud was generated as shown below. This final point cloud was the basis of the generation of the geometries of the billboards, road, and obstructions to be utilized for visibility analysis.

(a)

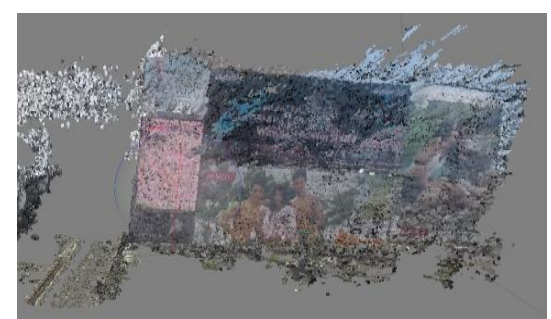

(b)

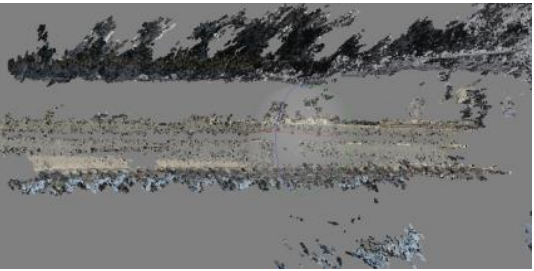

(c)

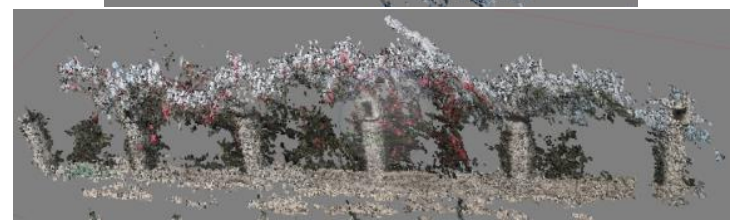

Figure 7. Generated point clouds of (a) billboards; (b) road; and (c) obstructions

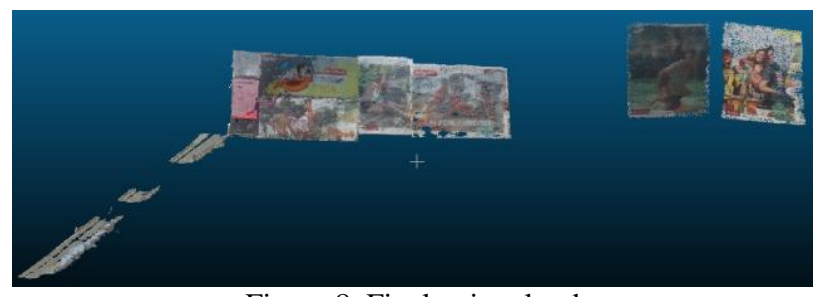

Figure 8. Final point cloud

\subsection{Billboard-road-obstruction geometry}

The next figure shows the billboard-road-obstructions vectorbased environment produced after digitizing the various sets of features. All of the billboards were digitized as rectangular. The road was represented by the gray rectangular figure and the obstructions were digitized as green irregularly-shaped figure. Seen in Figure 9 are the digitized road, billboards, and obstructions together with the corresponding 100 target points on each billboard and 100 observer points along the road. Similarly, the same environment is shown in Figure 10 but the target points for the logos are seen.

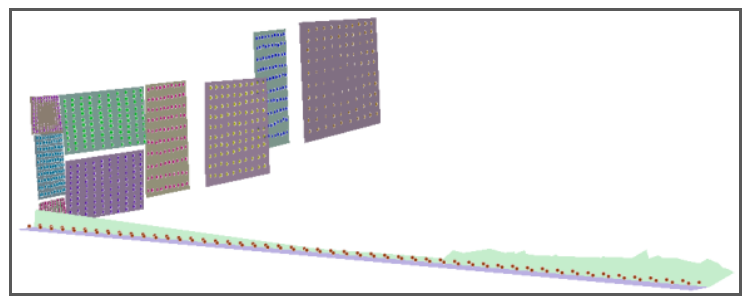

Figure 9. Billboard-road-obstruction environment with observer and target points

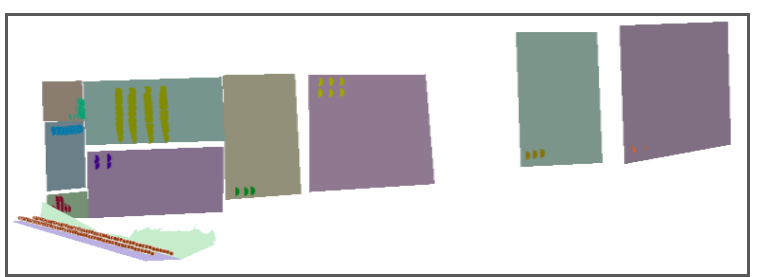

Figure 10. Road and billboards polygons with the varying number of target points on each billboard

\subsection{Assessment}

\subsubsection{Mean Percent Visibility}

Using equation 1, the Mean Percent Visibility for left, right, and both lanes were calculated. The table below summarizes the Mean Percent Visibility of each billboard as assessed along 
each lane and along the whole width of the two-lane road from the sets of observer points defined. The farther billboards relative to the road (Billboards 7-9), have lower MPV than billboards 1, 2, and 4 which are placed near the road. Moreover, these billboards have low MPV since their orientation is not directly facing the road of interest even though their size is bigger than the other billboards. In the case of billboards 3 and 5, even though they are placed relatively near the road, they attained a low MPV also because they are placed at a lower height as compared to other billboards; hence, they were completely blocked by the obstructions on the side of the road.

\begin{tabular}{|c|c|c|c|}
\hline Billboard & Left Lane & Right Lane & Both Lanes \\
\hline 1 & 97.32 & 95.90 & 96.61 \\
\hline 2 & 99.42 & 94.00 & 96.71 \\
\hline 3 & 30.00 & 52.08 & 41.04 \\
\hline 4 & 97.10 & 79.14 & 88.12 \\
\hline 5 & 57.66 & 38.86 & 48.26 \\
\hline 6 & 66.44 & 41.08 & 53.76 \\
\hline 7 & 5.86 & 74.04 & 39.95 \\
\hline 8 & 32.64 & 24.82 & 28.73 \\
\hline 9 & 34.74 & 25.22 & 28.98 \\
\hline \multicolumn{4}{|c}{ Table 2. Mean Percent Visibility } \\
\hline
\end{tabular}

\subsubsection{Visibility Raster of the Billboards}

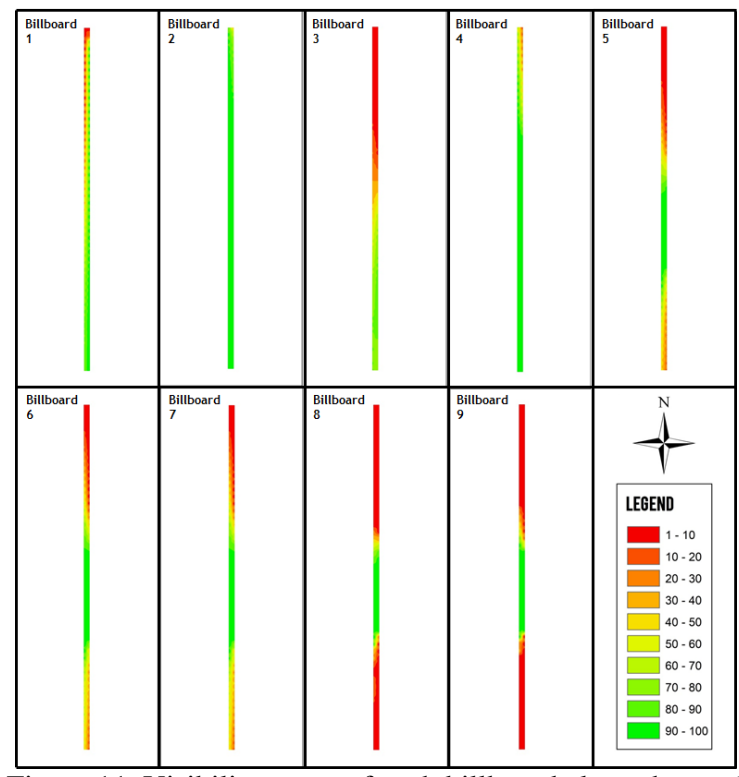

Figure 11. Visibility raster of each billboard along the road

Shown above are the road visibility raster representations of the nine billboards, placed side by side for comparison. It can be seen that the nearer billboards, with respect to the road, are more visible, in general, than those billboards that are located farther, with respect to the road. Although, billboards 6, 7, 8 and 9 are bigger in size than billboards $1,2,3$, and 4, they are placed at a more distant location; hence, they were blocked by the obstructions along the road - plants, bridge railings, and other nearer billboards.

The visibility raster representations also show that the obstructions were located at the beginning and at the end of the road. The middle part has relatively small amount of obstructions to none. This matched with the actual situation in Guadalupe bridge, northbound section. The obstructions are located at the start and at the end of the road, and few obstructions are placed at the middle part. Another reason for this is the placement of the billboards in relation to one another that these billboards may obstruct one another. This is evident in the visibility raster representations of Billboards $6,7,8$, and 9. These billboards were placed behind the Billboards 1, 2, 3, 4, and 5 and were being blocked when passing through the middle portion of the road.

\subsubsection{Mean Logo Percent Visibility (MLPV)}

The logo is the most important part of an advertisement, besides the fact that it informs the consumers of what brand is being endorsed in an advertisement, it also gives branding to a specific product. Therefore, the placement, sizing, and format, in general, of a logo shall be planned well to be effective and efficient of its purpose.

As seen in the Table 3, billboards 1 and 2 attained the $100 \%$ logo percent visibility while billboards 6,8 and 9 attained logo percent visibility lower than $30 \%$. These billboards were the larger ones, compared with the others. Possible explanation for this is the fact that these billboards, billboards 6, 8, and 9, despite their size, their logos only occupied small space on the billboards and are too small to be seen from the observer points and therefore, by the target market, which are the passengers in a moving vehicle.

\begin{tabular}{|c|c|}
\hline Billboards & MLPV \\
\hline 1 & 100.00 \\
\hline 2 & 100.00 \\
\hline 3 & 35.53 \\
\hline 4 & 90.11 \\
\hline 5 & 86.50 \\
\hline 6 & 28.33 \\
\hline 7 & 60.00 \\
\hline 8 & 22.67 \\
\hline 9 & 24.00 \\
\hline
\end{tabular}

Table 3. Mean Logo Percent Visibility (MLPV)

\subsubsection{Validation survey results}

The validation survey was participated by 40 respondents. The mean percent visibility was obtained by getting the mean of the answers of the respondents. Based on the results of the survey, which is shown in the table below, billboards 2, 4, 5, 6 and 7 attained high visibility percentages which are within the range of $71 \%$ to $79 \%$ while billboards $1,3,8$, and 9 attained low visibility percentages that are within the range of $39 \%$ to $52 \%$. Even though these percent visibilities from the survey results are subject to personal factors, the proportionality of the survey results with the actual results validate the MPV determined from the visibility analysis performed. For the majority of the survey results, the percent visibilities corresponded with the results from the visibility analysis performed with the exception of Billboard 1. Due to the limitations that survey respondents were just asked to watch the video traversing the road instead of asking them to pass through the road and observe the billboards, this disagreement of results in Billboard 1 is most likely due to the angle of placement of the camera during the acquisition of videos 1 \& 2 .

\begin{tabular}{|c|c|c|}
\hline Billboard & Actual Results & Survey Results \\
\hline 1 & 96.61 & 39.25 \\
\hline 2 & 96.71 & 73.50 \\
\hline 3 & 41.04 & 52.00 \\
\hline 4 & 88.12 & 78.75 \\
\hline
\end{tabular}




\begin{tabular}{|l|l|l|}
5 & 48.26 & 71.25 \\
\hline 6 & 53.76 & 71.00 \\
\hline 7 & 39.95 & 71.25 \\
\hline 8 & 28.73 & 49.50 \\
\hline 9 & 28.98 & 49.75 \\
\hline
\end{tabular}

Table 4. Comparison of MPV from analysis and survey

A similar comparison was performed to validate the MLPV. However, in this case, instead of asking percent visibilities from respondents, they were asked to identify the location of the logos from defined area divisions on a billboard. It can be clearly seen in Table 5 that the survey results contradicted the results from the visibility analysis. Even though logos were small and badly placed on a billboard, viewers still recognized them and identified their locations. This can be accounted for the product familiarity of the respondents. Since the similar product displayed on billboards 5-9 was a popular one in the country, they were able to remember its location as compared to other billboards whose logos were appropriately sized and space but were less popular and striking. Nonetheless, this study only focuses on the visibility of the billboards and their logos and cognitive analysis towards advertisements was not considered here.

\begin{tabular}{|c|c|c|}
\hline Billboard & Actual Results & Survey Results \\
\hline 1 & 100.00 & 32.50 \\
\hline 2 & 100.00 & 40.00 \\
\hline 3 & 35.53 & 42.50 \\
\hline 4 & 90.11 & 40.00 \\
\hline 5 & 86.50 & 35.00 \\
\hline 6 & 28.33 & 60.00 \\
\hline 7 & 60.00 & 32.50 \\
\hline 8 & 22.67 & 55.00 \\
\hline 9 & 24.00 & 50.00 \\
\hline
\end{tabular}

Table 5. Comparison of MLPV from analysis and survey

\section{CONCLUSIONS AND RECOMMENDATIONS}

\subsection{Conclusions}

This research aimed to test the application of structure-frommotion photogrammetry to assess the effectiveness of huge outdoor advertisements through visibility analysis. From the analyses performed, it was determined that the mean percent visibilities of the nine billboards as seen along the along Guadalupe Bridge in EDSA Highway ranged from $28.73 \%$ to $96.71 \%$. Moreover, the mean percent visibilities of the product logos on each of the billboards were also determined to assess if the logos were properly placed on the billboard. It was seen that 4 out of the nine billboards had a mean logo percent visibility less than 50\%--denoting them ineffective and bad logo placement in terms of their visibilities. These percent visibilities were also visualized through visibility raster representations per billboard.

A validation survey was conducted to confirm the analysis performed. For the most part, the validation confirmed the visibility analysis performed; however, due to limitations in the conduct of the validation survey itself as well as the disregard of the familiarity of the respondents to the area, the results varied for some billboards. In terms of the analysis of the logos, the validation survey did not confirm at all the results of the visibility analysis. This can be due to the prior knowledge and familiarity of the respondents to the area and the possible popularity and design of the logos of products observed.
The placements and orientation of the billboards as well as the placement of the logos are vital to the effectiveness of the billboards for advertising purposes. Through structure from motion photogrammetry, visibility analysis can be performed to various huge outdoor advertisements for advertisers to strategically identify prime locations for advertisements as well as the design and placements of its contents.

\subsection{Recommendations}

This research focused and relied on the method of data acquisition from handheld cameras only. The researchers recommend using additional methods on acquiring data such UAV to produce a denser point cloud of the environment for a better visibility analysis. As for other concerns, the researchers also recommend using other types of vehicles which may result to different standard height of eye level of a passenger. Moreover, since this study only considered the two rightmost lanes from the 5-lane road on the bridge, the researchers would like to recommend getting the visibility of each billboards on the rest, leftmost lanes of the road. Also, since there are other roads in Guadalupe, e.g. oriented perpendicular to the northbound of EDSA, these could be also considered to compare visibilities of the billboards because these billboards can also be seen from that point of view.

This research could be further improved by looking on the valuation aspect of the billboards. In the assessment of the effectiveness of the billboards, the costing for ad placement is not only in terms of size but should also consider the placements and orientations are factors.

\section{REFERENCES}

Lithgow, D. (1999). The Effective Use of Billboards as a Communications Tool. Web.

Lucas, J. \& Prensky, D. (1997). Evaluating the Effectiveness of Place-based Media. Measuring Advertising Effectiveness. Wells, W. (Ed.) New Jersey: Lawrence Erlbaum Associates, Inc.

Gudis, C. (2004). Chapter 5: Modern Art and Photogrammetry. Buyways (pp 79-98). NY: Routledge

C. Ayd $\tilde{A}, \hat{A} \pm n, \quad$ Cevdetx \& Recep, Nisanc $\tilde{A}, \hat{A} \pm . \quad$ (2008). Environmental Harmony and Evaluation of Advertisement Billboards with Digital Photogrammetry Technique and GIS Capabilities: A Case Study in the City of Ankara. Sensors. 8. $10.3390 / \mathrm{s} 8053271$

Chmielewski, Szymon \& Tompalski, Piotr. (2017). Estimating outdoor advertising media visibility with voxel-based approach. Applied Geography. 87. 1-13. 10.1016/j.apgeog.2017.07.007.

Chmielewski, Szymon \& Lee, Danbi. (2015). GIS-based 3D Visibility Modelling of Outdoor Advertising in Urban Areas. 10.5593/SGEM2015/B22/S11.116.

Moulon, Pierre \& Monasse, Pascal \& Marlet, Renaud. (2012). Adaptive Structure from Motion with a Contrario Model Estimation. 11th Asian Conference on Computer Vision - ACCV. 10.1007/978-3-642-37447-0_20.

CloudCompare Project Team, 2015. CloudCompare Software. www.cloudcompare.org(20 September 2017).

Revised August 2019 